\title{
Structural and biochemical analyses of PadR-mediated transcriptional regulation
}

\author{
Sun Cheol Park ${ }^{1}$, Yun Mi Kwak ${ }^{1}$, Wan Seok Song ${ }^{1}$, Minsun Hong ${ }^{2}$ and Sung-il Yoon ${ }^{1 *}$ \\ ${ }^{1}$ Division of Biomedical Convergence, College of Biomedical Science, Kangwon National University, \\ Chuncheon 24341, Republic of Korea, ${ }^{2}$ Division of Biological Science and Technology, Yonsei University, \\ Wonju 26493, Republic of Korea.
}

\begin{abstract}
Microorganisms recognize and respond to harmful external environments by producing defense proteins, the expression of which is regulated by environment-sensing transcriptional regulators. PadR is a bacterial transcription factor that belongs to the PadR family and negatively controls the expression of phenolic acid decarboxylase, which detoxifies harmful phenolic acids. Although PadR family members have been shown to be involved in toxin expression, antibiotic resistance, and carbon catabolism, it is unknown how they sense environmental factors and regulate transcription. To reveal the structural mechanism whereby PadR detects its effector, phenolic acid, and regulates gene expression, we have determined the crystal structures of Bacillus subtilis PadR in complex with phenolic acid or operator dsDNA. PadR contains two domains, namely, an Nterminal winged helix-turn-helix domain (NTD) and a C-terminal homodimerization domain (CTD) and assembles into a dimer. PadR forms an interdomain pocket between the NTD and the CTD and uses the pocket to directly interact with a phenolic acid effector molecule. The PadR dimer symmetrically recognizes the palindromic sequence of the operator DNA using the two NTDs. Furthermore, our mutational and biochemical analyses demonstrated that PadR Tyr20, Tyr42, and Lys67 play a critical role in DNA binding energetics. Based on structural comparison, we propose that PadR mediates transcriptional regulation in response to phenolic acid through allosteric interdomain-reorganization mechanism.
\end{abstract}

